\title{
Review Article \\ The Relationship between Betatrophin Levels in Blood and T2DM: A Systematic Review and Meta-Analysis
}

\author{
Song Yue, Jingyang Wu, ${ }^{1}$ Jiahua Zhang, ${ }^{1}$ Lei Liu, ${ }^{1,2}$ and Lei Chen ${ }^{1}$ \\ ${ }^{1}$ Department of Ophthalmology, The First Affiliated Hospital of China Medical University, Shenyang 110001, China \\ ${ }^{2}$ Department of Epidemiology, School of Public Health, China Medical University, Shenyang 110001, China \\ Correspondence should be addressed to Lei Liu; liuleijiao@163.com and Lei Chen; leichen0501@163.com
}

Received 21 December 2015; Revised 1 March 2016; Accepted 16 March 2016

Academic Editor: Michele Malaguarnera

Copyright (c) 2016 Song Yue et al. This is an open access article distributed under the Creative Commons Attribution License, which permits unrestricted use, distribution, and reproduction in any medium, provided the original work is properly cited.

\begin{abstract}
Background. In order to clarify previous ambiguous research conclusions, a meta-analysis was made to investigate the relationship between betatrophin levels in blood and type 2 diabetes mellitus (T2DM). Methods. We have searched all the English and Chinese references regarding the relationship between betatrophin and diabetes in database both manually and online. Strict criteria have been established to include and exclude articles, with Mean and Standard Deviation as statistics to evaluate strength of association. We have chosen either fixed- or random effect model according to heterogeneity inspection results and used Begg's test and Egger's test to analyze publication bias. Results. A total of 11 studies were included in this meta-analysis. Meta-analysis indicated a significant association between betatrophin and T2DM (Mean: 329.46; 95\% confidence interval: 182.51 to $476.42, P<0.0001$ ). However, in the subgroup analysis, there was no significant statistic between betatrophin concentration and T2DM within Caucasian population (Mean: 98.40 ; $95 \%$ confidence interval: -1585.08 to $1781.88, P=0.91$ ). Conclusions. Such relationship may suggest preference for association between betatrophin and T2DM in different population.
\end{abstract}

\section{Introduction}

Diabetes mellitus (DM) is a type of metabolic disease characterized by hyperglycemia. It is caused by either defected insulin secretion or damaged biological function, or both. The statement of a high-level blood glucose that a body is in for a long time will lead to dysfunction of a variety of tissues. Nowadays, such methods as taking antidiabetic medicines or injecting insulin to cope with diabetes are the usual practice, but there is no way of thorough treatment. Recently, researchers [1] from Harvard Stem Cell Institute (HSCI) found a new hormone and named it betatrophin, which, closely related to diabetes treatment, could increase the quantity of cells that produce insulin in mice quickly. Betatrophin, mainly circulated in liver and adipose tissues, promotes greatly the proliferation of pancreatic beta cells, plays an important role in modulating glycolipid metabolism, and maybe replaces insulin in the effective treatment of diabetes. Before that, different research teams name it differently according to its different functions, including angiotensin amyloid 8 (ANGPTL8) [2], lipoprotein lipase inhibition
(Lipasin) [3], and refeeding induced in fat and liver (RIFL) [4]. Previous reports [5] showed that serum betatrophin levels were significantly higher in T2DM, while others showed that the expression of betatrophin index declined [6], and the third conclusion was that there was no statistical significance on the relationship between betatrophin levels and T2DM [7]. In order to evaluate the correlation between betatrophin and diabetes objectively, we perform a meta-analysis on related studies that are qualified, in the hope of getting a more persuasive conclusion.

\section{Methods}

2.1. Selection of Eligible Studies. Two researchers (Yue and $\mathrm{Wu}$ ) have conducted document retrieval to the relationship between betatrophin and DM independently and elaborately, who have searched such database in the text as PubMed, MEDLINE, Embase, Cochrane Library with "betatrophin and diabetes," "Angptl8 and diabetes," "RIFL and diabetes," and "Lipasin and diabetes" combined and searched others including CNKI, China Wan-Fang database, and Chongqing 
VIP database with "betatrophin and diabetes." The latest search date is May 1, 2015. We have studied every article selected as "Related Articles" by PubMed and searched it to get extra potentially related articles. We have also searched the references and contacted the authors to get extra articles. When there was ambiguity about the results or lack of sufficient data, we contacted all authors to make it clear. The searching method has been made beyond linguistic limits, but we only included the articles published in English or Chinese language.

2.2. Selection Criteria. The following criteria have to be met for the articles to be included: (1) case-control study; (2) the cases in those studies that were type 2 diabetes; (3) all the case groups which were in accordance with international criteria for diagnosis of type 2 diabetes; (4) articles published as papers until May 2015; (5) articles that provided directly or indirectly the results on relevant research index in case group and control group.

2.3. Exclusion Criteria. Exclusion criteria are defined as follows: (1) articles in which the case group was diagnosed with type 1 diabetes; (2) research with insufficient data; (3) research with data that could not be converted; (4) adopting the best-quality among research papers that were duplicated, repeatedly collected, or with similar data.

2.4. Data Extraction. The two researchers entered into each database, respectively, with standard procedures and extracted research data independently. If there was a discrepancy, the researchers would appraise the data together. The following information was gathered including the first author, publication year, nationality, blood sample, experimental method, sample size of patients and the control group, and Mean and Standard Deviation (SD) (part of the data were converted) of betatrophin levels.

The review and analysis were guided to conduct by the PRISMA statement for preferred reporting of meta-analysis [16].

2.5. Statistical Analysis. The RevMan5.3 statistical software provided by The Cochrane Collaboration was applied, with Mean and SD as statistics to evaluate strength of association. According to heterogeneity inspection results, corresponding pooled method was chosen: when there was no significant heterogeneity in research results $\left(I^{2}<50 \%\right)$, the fixed effect model was used to weight and pool the effect size. However, when research results appeared to be heterogeneous $\left(I^{2}>50 \%\right)$, the random effect model was adapted to weight and pool the effect size, and meta-analysis forest plot was drawn. We used statistical software named Comprehensive Meta-Analysis (http://www.meta-analysis .com/pages/demo.php) to draw funnel plots, and an asymmetrical one suggested the existence of heterogeneity. Besides, the researchers used Begg's and Egger's test to analyze publication bias, and it is considered that if $P<0.05$ in Begg's and Egger's test, publication bias was significant statistically. All $P$ values were two sided.

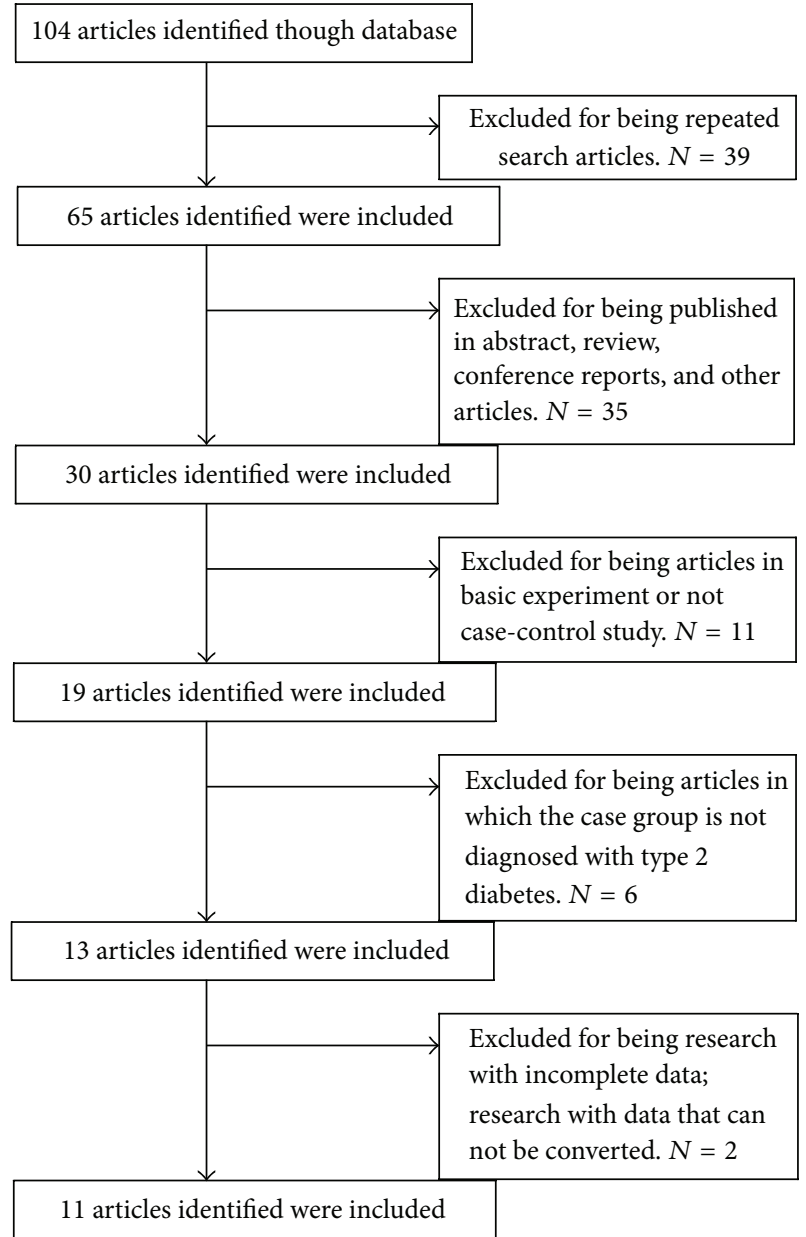

Figure 1: Flowchart demonstrating those studies that were processed for inclusion in the meta-analysis.

\section{Results}

3.1. Study Inclusion and Characteristics. After the initial screening, there were 104 papers which reached screening stage including 71 in English and 33 in Chinese. After strict selection process, a total of 11 articles [5-15] remained for this meta-analysis. The results of the selection process were displayed in Figure 1. There were nine English literatures and two Chinese publications with 490 cases and 508 controls. The test samples were plasma in two literatures and serum in the rest nine, and all the test methods in included literatures were enzyme-linked immunosorbent assay (ELISA) method. The characteristics and findings of these experiments were presented in Table 1.

3.2. Outcomes. All this meta-analysis outcomes were shown in Figures 2 and 3. (1) It is obvious that heterogeneity existed in all the eleven included papers $\left(P<0.00001, I^{2}=99 \%\right)$. Therefore, the random effect model was employed to conduct pooled analysis. The outcomes of pooled Mean $[95 \% \mathrm{CI}]$ at $329.46[182.51,476.42], Z=4.39$, showed that the circulating level of betatrophin in T2DM patient blood was higher than that in control groups, in Figure 2(a). 
TABLE 1: Characteristics of eligible studies included in the meta-analysis.

\begin{tabular}{|c|c|c|c|c|c|c|c|c|}
\hline \multirow[b]{2}{*}{ First author } & \multirow[b]{2}{*}{ Publication year } & \multirow[b]{2}{*}{ Country } & \multirow[b]{2}{*}{ Sample } & \multirow[b]{2}{*}{ Methods } & \multicolumn{2}{|c|}{ Cases } & \multicolumn{2}{|c|}{ Controls } \\
\hline & & & & & Subjects $(n)$ & $\begin{array}{c}\text { Betatrophin } \\
(\text { Mean } \pm \mathrm{SD}, \mathrm{pg} / \mathrm{mL})\end{array}$ & Subjects $(n)$ & $\begin{array}{c}\text { Betatrophin } \\
(\text { Mean } \pm \mathrm{SD}, \mathrm{pg} / \mathrm{mL})\end{array}$ \\
\hline Fenzl [7] & 2014 & Germany & Plasma & ELISA & 18 & $1646.5 \pm 551.12$ & 19 & $1643.4 \pm 1002.11$ \\
\hline Chen [8] & 2014 & China & Serum & ELISA & 112 & $798.6 \pm 449.78$ & 137 & $692.7 \pm 339.44$ \\
\hline Espes [9] & 2014 & Sweden & Plasma & ELISA & 27 & $893 \pm 415.69$ & 18 & $639 \pm 280.01$ \\
\hline $\mathrm{Fu}[10]$ & 2014 & USA & Serum & ELISA & 14 & $5560 \pm 2731.41$ & 15 & $2190 \pm 929.52$ \\
\hline $\mathrm{Hu}[5]$ & 2014 & China & Serum & ELISA & 83 & $613.08 \pm 65.15$ & 83 & $296.57 \pm 52.16$ \\
\hline Gómez-Ambrosi [6] & 2014 & Spain & Serum & ELISA & 15 & $13500 \pm 8800$ & 33 & $45100 \pm 24400$ \\
\hline Wang [11] & 2015 & China & Serum & ELISA & 35 & $1123.52 \pm 319.01$ & 31 & $669.15 \pm 318.16$ \\
\hline Xie [12] & 2014 & China & Serum & ELISA & 50 & $412.53 \pm 237.67$ & 50 & $306.20 \pm 283.82$ \\
\hline Yamada [13] & 2015 & Japan & Serum & ELISA & 30 & $1614 \pm 647$ & 12 & $300 \pm 236$ \\
\hline Guo [14] & 2015 & China & Serum & ELISA & 56 & $162.56 \pm 42.25$ & 60 & $123.52 \pm 28.19$ \\
\hline Xie [15] & 2015 & China & Serum & ELISA & 50 & $833.45 \pm 456.21$ & 50 & $541.68 \pm 136.2$ \\
\hline
\end{tabular}

TABLE 2: All the forest plot data summary.

\begin{tabular}{lcccccc}
\hline Subgroup & Article number & Total $(95 \%$ CI $)$ & $I^{2}$ & $Z$ & $P$ & Model \\
\hline Chinese & 6 & $214.15[57.65,370.65]$ & $99 \%$ & 2.68 & $P=0.007$ & Random \\
Caucasian & 4 & $98.40[-1585.08,1781.88]$ & $95 \%$ & 0.11 & $P=0.91$ & Random \\
Plasma & 2 & $220.47[31.27,409.67]$ & 0 & 2.28 & $P=0.02$ & Fixed \\
Serum & 9 & $358,64[198.04,519.24]$ & $99 \%$ & 4.38 & $P<0.0001$ & Random \\
Total & 11 & $329.46[182.51,476.42]$ & $99 \%$ & 4.39 & $P<0.0001$ & Random \\
\hline
\end{tabular}

(2) A subgroup analysis was carried out based on different group of people. The results showed that heterogeneity $(P<$ $0.00001, I^{2}=99 \%$ ) existed in the six papers that performed the study on Chinese people and therefore the random effect model was used to do meta-analysis. The results of pooled Mean [95\% CI] at $214.15[57.65,370.65], Z=2.68$, revealed in Figure 2(b) that the circulating level of betatrophin in Chinese T2DM patient blood was higher than that in control groups. Likewise, heterogeneity $\left(P<0.00001, I^{2}=95 \%\right)$ was more significant in the four papers that studied Western Caucasians and therefore the random effect model was used to do pooled analysis. As the result, pooled Mean [95\% CI] was at $98.40[-1585.08,1781.88], Z=0.11$, which showed that the index of betatrophin circulating level in Caucasians' blood had no statistical significance, in Figure 2(c).

(3) Another subgroup analysis was made based on different types of blood samples. The two papers with plasma as research sample did not present a conclusion of heterogeneity $\left(P=0.38, I^{2}=0\right)$, and the fixed effect model was chosen to do pooled analysis. The results of pooled Mean (95\% CI) at 220.47 [31.27, 409.67], $Z=2.28$, revealed in Figure 3(a) that the plasma concentrations of betatrophin are higher in T2DM patient.

In addition, the random effect model was carried out to do pooled analysis according to the result of heterogeneity $\left(P<0.00001, I^{2}=99 \%\right)$ in the nine papers with serum as research sample. The results of pooled Mean (95\% CI) at 358.64 [198.04,519.24], $Z=4.38$, revealed in Figure 3(b) that the serum concentrations of betatrophin are also higher in T2DM patient. All the forest plot data summary was in Table 2.

According to Begg's and Egger's tests (Begg, $P=0.11$; Egger, $P=0.25)$, the funnel plot was not asymmetrical (Figure 4), which demonstrated a nonsignificant $P$ value publication bias.

\section{Discussion}

$\mathrm{DM}$ is a metabolic disorder caused by pancreatic beta cells defect or damage [17], characterized by increased chronic hyperglycemia level. Long-term hyperglycemia can cause damage to multiple systems [18]. To date, main therapeutic approach in treating diabetes is to improve insulin resistance, promote insulin secretion, or preserve the remaining beta cell function by using insulin or drugs [19]. Although it can control the blood glucose to some extent, it cannot solve the fundamental problem: relative or absolute pancreatic beta cells deficiency.

Previous report showed that betatrophin-encoded protein could significantly promote the proliferation of mouse pancreatic beta cells with increasing number so as to enhance glucose tolerance. With these striking study findings, many scholars conducted research on this newly discovered peptide and examined the relevance between betatrophin and T2DM by detecting the circulating levels of betatrophin in T2DM patients. However, the conclusions were contradictory. 


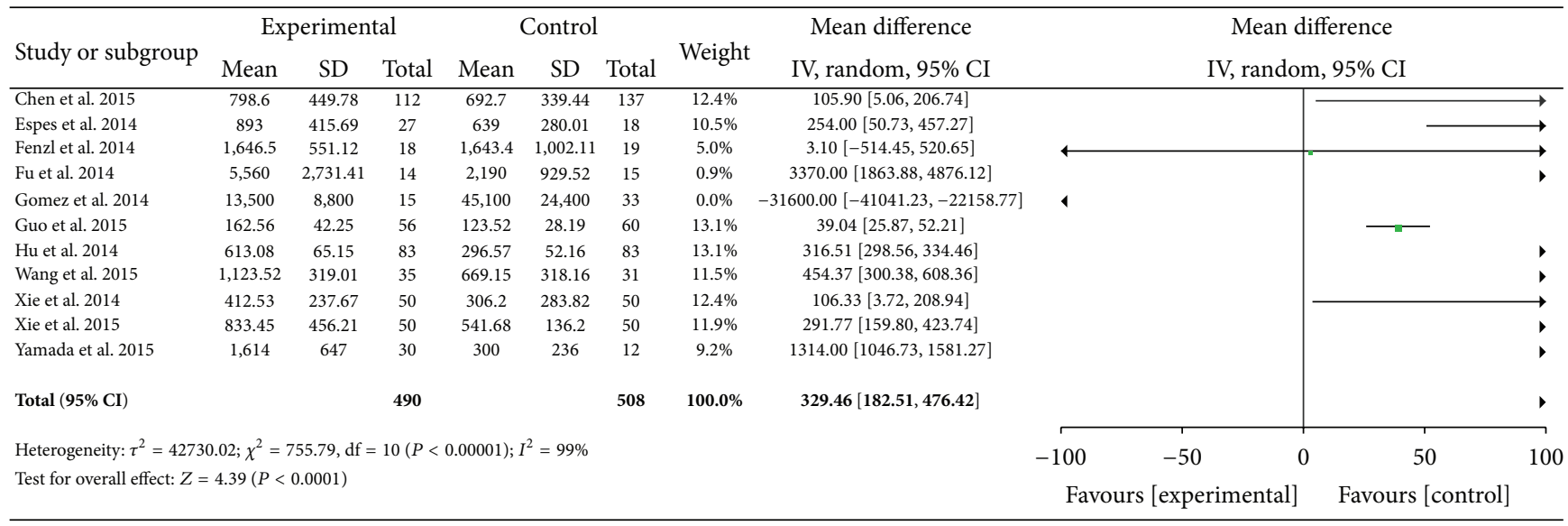

(a)

\begin{tabular}{|c|c|c|c|c|c|c|c|c|c|c|c|c|c|}
\hline \multirow{3}{*}{$\begin{array}{l}\text { Study or subgroup } \\
\text { Chen et al. } 2015\end{array}$} & \multicolumn{3}{|c|}{ Experimental } & \multicolumn{3}{|c|}{ Control } & \multirow{2}{*}{ Weight } & \multirow{2}{*}{$\begin{array}{c}\text { Mean difference } \\
\text { IV, random, 95\% CI }\end{array}$} & \multirow{2}{*}{\multicolumn{5}{|c|}{$\begin{array}{c}\text { Mean difference } \\
\text { IV, random, 95\% CI }\end{array}$}} \\
\hline & \multirow{2}{*}{$\begin{array}{c}\text { Mean } \\
798.6\end{array}$} & \multirow{2}{*}{$\begin{array}{c}\mathrm{SD} \\
449.78\end{array}$} & \multirow{2}{*}{$\frac{\text { Total }}{112}$} & \multirow{2}{*}{$\begin{array}{c}\text { Mean } \\
692.7\end{array}$} & \multirow{2}{*}{$\frac{\mathrm{SD}}{339.44}$} & \multirow{2}{*}{$\frac{\text { Total }}{137}$} & & & & & & & \\
\hline & & & & & & & $16.6 \%$ & $105.90[5.06,206.74]$ & & & & & $\vec{\longrightarrow}$ \\
\hline Espes et al. 2014 & 893 & 415.69 & 27 & 639 & 280.01 & 18 & $0.0 \%$ & $254.00[50.73,457.27]$ & & & & & \\
\hline Fenzl et al. 2014 & $1,646.5$ & 551.12 & 18 & $1,643.4$ & $1,002.11$ & 19 & $0.0 \%$ & $3.10[-514.45,520.65]$ & & & & & \\
\hline Fu et al. 2014 & 5,560 & $2,731.41$ & 14 & 2,190 & 929.52 & 15 & $0.0 \%$ & $3370.00[1863.88,4876.12]$ & & & & & \\
\hline Gomez et al. 2014 & 13,500 & 8,800 & 15 & 45,100 & 24,400 & 33 & $0.0 \%$ & $-31600.00[-41041.23,-22158.77$ & & & & & \\
\hline Guo et al. 2015 & 162.56 & 42.25 & 56 & 123.52 & 28.19 & 60 & $17.8 \%$ & $39.04[25.87,52.21]$ & & & & $\longrightarrow$ & \\
\hline Hu et al. 2014 & 613.08 & 65.15 & 83 & 296.57 & 52.16 & 83 & $17.8 \%$ & $316.51[298.56,334.46]$ & & & & & • \\
\hline Wang et al. 2015 & $1,123.52$ & 319.01 & 35 & 669.15 & 318.16 & 31 & $15.2 \%$ & $454.37[300.38,608.36]$ & & & & & • \\
\hline Xie et al. 2014 & 412.53 & 237.67 & 50 & 306.2 & 283.82 & 50 & $16.6 \%$ & $106.33[3.72,208.94]$ & & & & & $\rightarrow$ \\
\hline Xie et al. 2015 & 833.45 & 456.21 & 50 & 541.68 & 136.2 & 50 & $15.9 \%$ & $291.77[159.80,423.74]$ & & & & & • \\
\hline Yamada et al. 2015 & 1,614 & 647 & 30 & 300 & 236 & 12 & $0.0 \%$ & $1314.00[1046.73,1581.27]$ & & & & & \\
\hline Total $(95 \% \mathrm{CI})$ & & & 386 & & & 411 & $100.0 \%$ & $214.15[57.65,370.65]$ & & & & & - \\
\hline Heterogeneity: $\tau^{2}=3567$ & $.72 ; \chi^{2}=6$ & 18.95, $\mathrm{df}=$ & $5(P<0$ & 0001); I & $99 \%$ & & & & -100 & -50 & 0 & 50 & 100 \\
\hline Test for overall effect: $Z=$ & $2.68(P=0$ & .007) & & & & & & & Fav & [exper & & Favours [ & \\
\hline
\end{tabular}

(b)

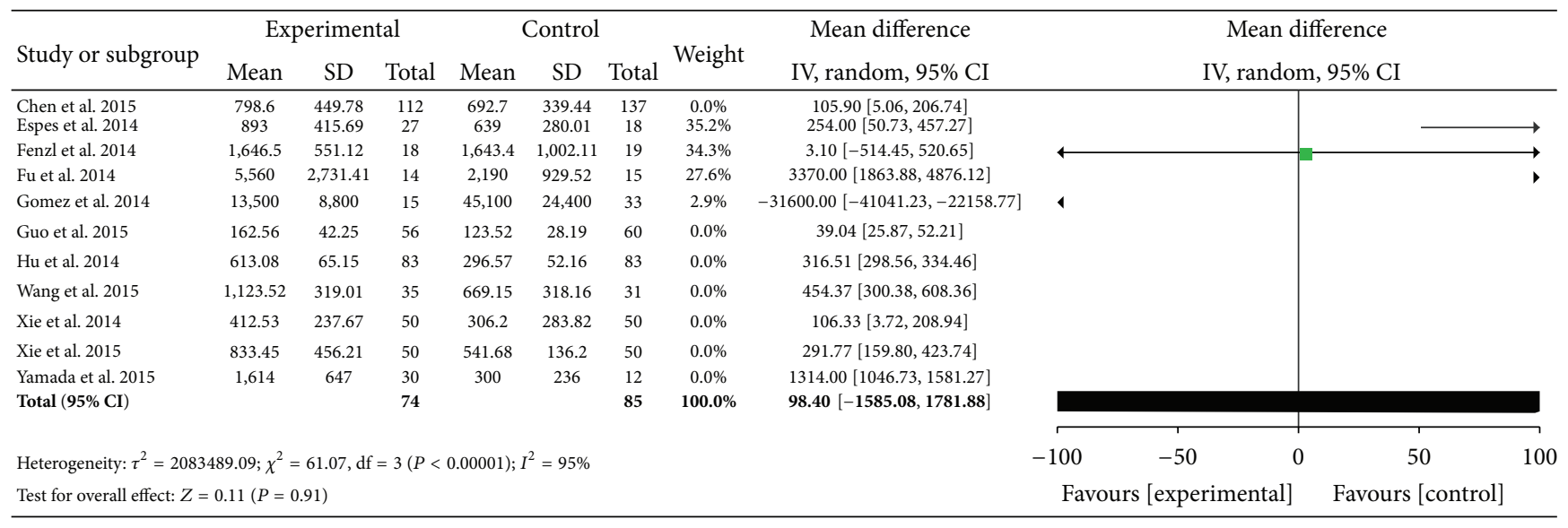

(c)

FIGURE 2: (a) Forest plot of the circulating level of betatrophin in T2DM patient, studies are pooled with random-effects model. (b) Forest plot of the circulating level of betatrophin in Chinese T2DM patient, studies are pooled with random-effects model. (c) Forest plot of the circulating level of betatrophin in Caucasians T2DM patient, studies are pooled with random-effects model.

This meta-analysis showed that the pooled value of Mean [95\% CI] was of statistical significance, revealing increased circulating levels of betatrophin in T2DM. By subgroup analyses, (1) all the research samples for Chinese people were serum, and the results showed that betatrophin circulating level increased in the serum of Chinese T2DM patients; (2) betatrophin circulating level increased in the plasma of the T2MD patients. Since the study subjects are all Caucasian, we 


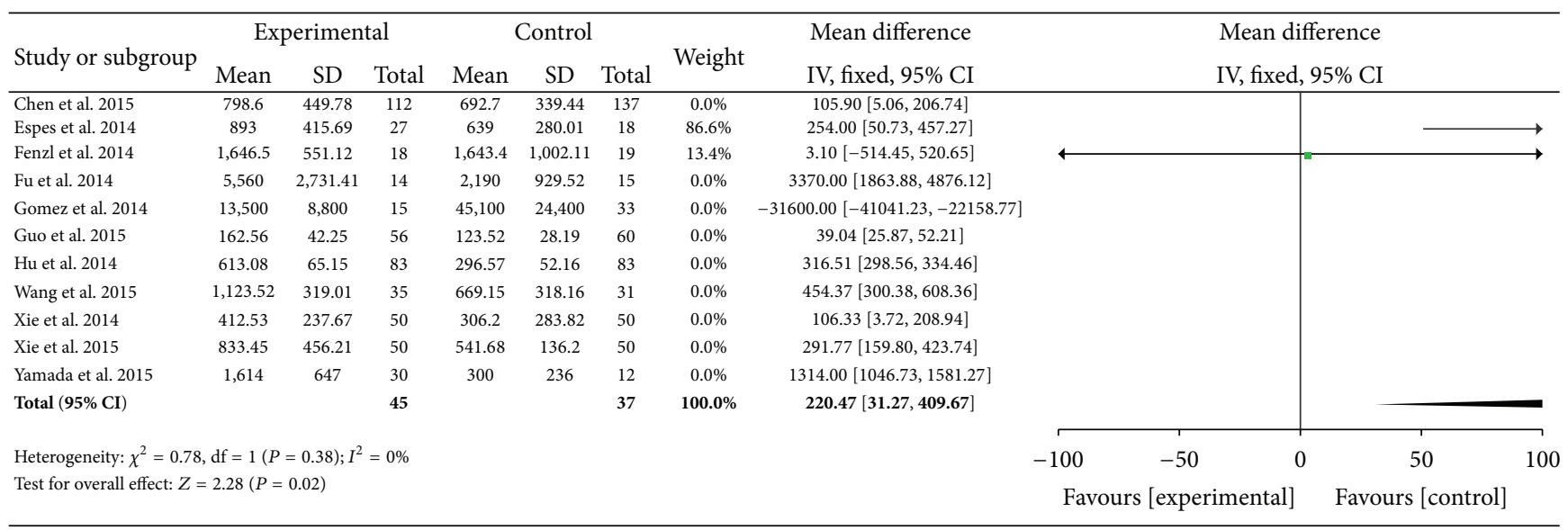

(a)

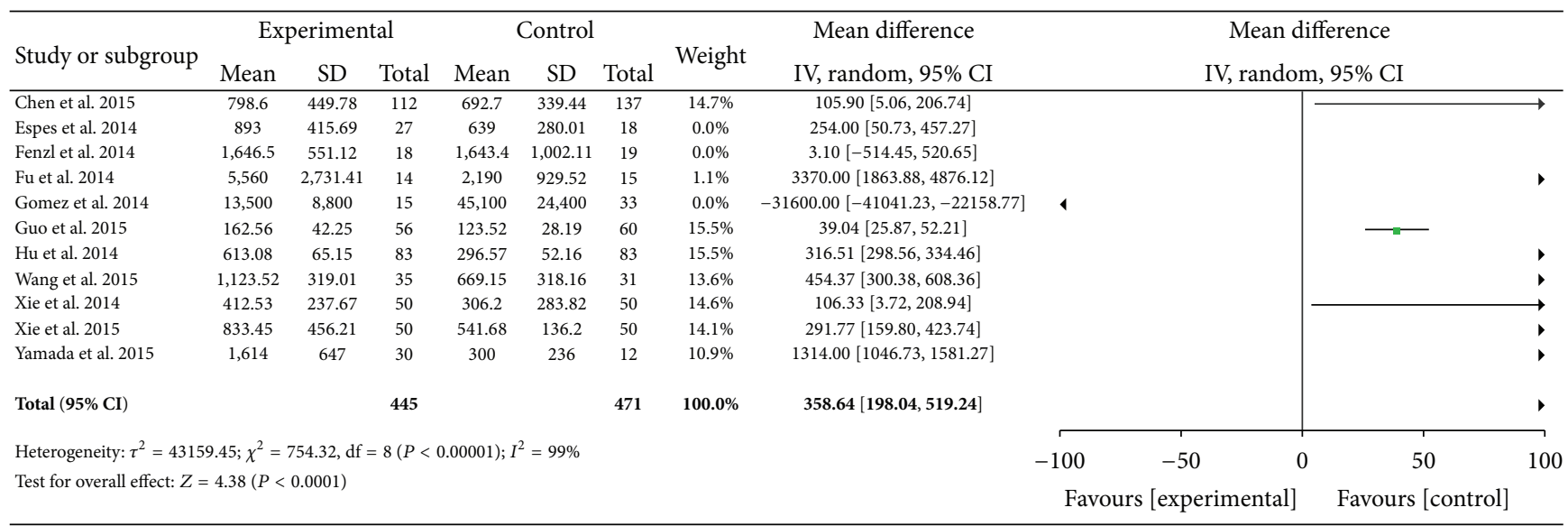

(b)

FIGURE 3: (a) Forest plot of the circulating level of betatrophin in T2DM patient plasma. (b) Forest plot of the circulating level of betatrophin in T2DM patient serum.

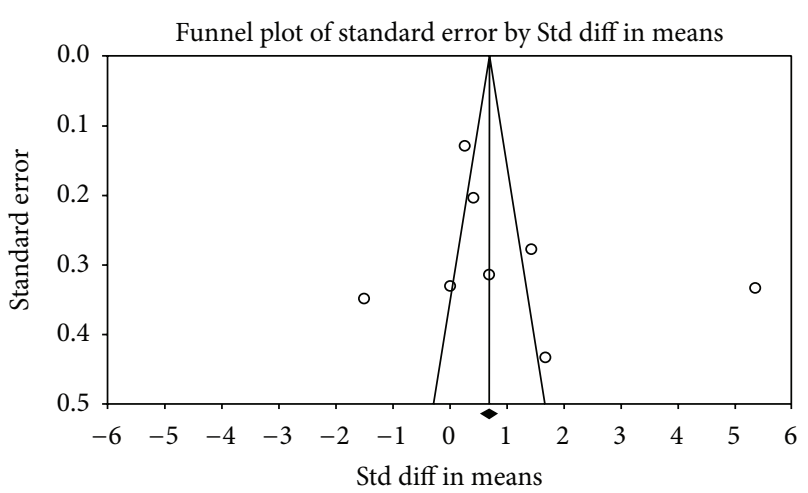

FIGURE 4: Funnel plot based on 11 case-control studies.

could make a conclusion for the moment: increased plasma betatrophin circulating levels in Caucasian T2DM patients.

However, the result showed that circulating levels of betatrophin in Caucasian T2DM patients have no statistical significance. There are differences between the result of this subgroup analyses and the overall result. Nevertheless, the results were consistent with the conclusions by Fenzl et al. [7]. In previous research [7], plasma betatrophin concentrations did not differ between patients with type 2 diabetes and nondiabetic controls. However, there were no sufficient theories to illustrate these results. So the exact mechanisms of betatrophin action in diabetic disease are still needed.

During the screening, Ebert et al. [20] also consider that betatrophin will increase in the serum of T2DM patients. However, the result of the article was presented by Median [interquartile range] and it cannot be converted into Mean \pm $\mathrm{SD}$, so we have to exclude it.

In this meta-analysis, the entire included case group is T2DM patients. Since the pathogenesis of T1DM is different from that of T2DM, we have not included articles on T1DM research. There have been scholars who have explored, among which Espes et. al. [21] studied betatrophin circulating level in the plasma of the Swedes. The study result showed that betatrophin circulating level in the serum of the T1DM patients is as twice as the normal glucose tolerance group $(300 \mathrm{pg} / \mathrm{mL})$. The article has also mentioned that betatrophin circulating level changes with different age groups in the healthy groups. More efforts are encouraged to explore this association. 
According to the forest plot A, substantial heterogeneity $\left(I^{2}=99 \%\right)$ was observed among the studies. To find the source of heterogeneity, sensitivity analysis was performed. Articles were excluded one by one before reanalyzing statistically. The results showed that there was always substantial heterogeneity. Heterogeneity increases were caused by selection bias existing in case group. The second was the small sample size. Different race and different sample are also the reasons of heterogeneity, because of which we conduct subgroup analyses. Fu et al's [22] research claims that different research results about the betatrophin circulating level in T2DM patients are caused by different ELISA research kits used and it may be also the reason of heterogeneity. A nonsignificant $P$ value according to Begg's and Egger's tests (Begg, $P=0.11$ Egger, $P=0.25$ ).

This is meta-analysis investigating the association between betatrophin and DM, which has significantly increased the statistical power. However, the present results of meta-analysis have some limitations. First, DM is a kind of disease influenced by multiple factors and there are complex interactions between them. In this meta-analysis we have not enough data to evaluate the interaction of betatrophin in diabetes and other factors. Second, we have no access to the data that are not published, so the publication bias cannot be avoided absolutely. Third, our search languages are only English and Chinese and research data of other races may have influence on the results. Fourth, we did not get the original data of the included literature, so we cannot guarantee the accuracy of the data.

Despite the limitations above, we believe that based on the positive results of the meta-analysis, it is worth for more scholars making further study in prospective study and follow-up research.

In conclusion, the meta-analysis of all published casecontrol studies on betatrophin and T2DM revealed increased circulating levels of betatrophin in patients with type 2 diabetes.

\section{Competing Interests}

The authors declare no competing financial interests.

\section{Authors' Contributions}

Song Yue and Jingyang Wu have contributed to the design of the study and analysis and interpretation of data and prepared all figures and tables. Song Yue, Jingyang Wu, Lei Liu, and Lei Chen drafted a part of the paper. Song Yue, Jiahua Zhang, and Jingyang Wu took part in analyzing data and drafting a part of the paper. All authors reviewed the paper.

\section{Acknowledgments}

The authors thank Miss Hu-nan in Dalian University of Foreign Languages for English language edition. This study was supported by National Natural Science Foundation of China (81300783) and Important Platform of Science and Technology for the University in Liaoning Province (16010).

\section{References}

[1] P. Yi, J.-S. Park, and D. A. Melton, "Betatrophin: a hormone that controls pancreatic $\beta$ cell proliferation," Cell, vol. 153, no. 4, pp. 747-758, 2013.

[2] F. Quagliarini, Y. Wang, J. Kozlitina et al., "Atypical angiopoietin-like protein that regulates ANGPTL3," Proceedings of the National Academy of Sciences of the United States of America, vol. 109, no. 48, pp. 19751-19756, 2012.

[3] R. Zhang, "Lipasin, a novel nutritionally-regulated liverenriched factor that regulates serum triglyceride levels," Biochemical and Biophysical Research Communications, vol. 424, no. 4, pp. 786-792, 2012.

[4] G. Ren, J. Y. Kim, and C. M. Smas, "Identification of RIFL, a novel adipocyte-enriched insulin target gene with a role in lipid metabolism," American Journal of Physiology-Endocrinology and Metabolism, vol. 303, no. 3, pp. E334-E351, 2012.

[5] H. Hu, W. Sun, S. Yu et al., "Increased circulating levels of betatrophin in newly diagnosed type 2 diabetic patients," Diabetes Care, vol. 37, no. 10, pp. 2718-2722, 2014.

[6] J. Gómez-Ambrosi, E. Pascual, V. Catalán et al., "Circulating betatrophin concentrations are decreased in human obesity and type 2 diabetes," The Journal of Clinical Endocrinology and Metabolism, vol. 99, no. 10, pp. E2004-E2009, 2014.

[7] A. Fenzl, B. K. Itariu, L. Kosi et al., "Circulating betatrophin correlates with atherogenic lipid profiles but not with glucose and insulin levels in insulin-resistant individuals," Diabetologia, vol. 57, no. 6, pp. 1204-1208, 2014.

[8] X. Chen, P. Lu, W. He et al., "Circulating betatrophin levels are increased in patients with type 2 diabetes and associated with insulin resistance," The Journal of Clinical Endocrinology \& Metabolism, vol. 100, no. 1, pp. E96-E100, 2015.

[9] D. Espes, M. Martinell, and P. O. Carlsson, "Increased circulating betatrophin concentrations in patients with type 2 diabetes," International Journal of Endocrinology, vol. 2014, Article ID 323407, 6 pages, 2014.

[10] Z. Fu, F. Berhane, A. Fite, B. Seyoum, A. B. Abou-Samra, and R. Zhang, "Elevated circulating lipasin/betatrophin in human type 2 diabetes and obesity," Scientific Reports, vol. 4, article 5013, 2014.

[11] Y. Y. Wang, D. Zhang, Z. R. Jiang, X. Q. Lu, and X. G. Wang, "The relationship between serum betatrophin levels of newly diagnosed type 2 diabetes patients and insulin resistance," China Modern Doctor, vol. 53, no. 3, pp. 4-6, 2015.

[12] X. M. Xie, T. Gao, M. L. Yang et al., "Betatrophin is related with Irisin in normal glucose tolerance people rather than newlydiagnosed type 2 diabetes mellitus patients," Shanghai Medical Journal, vol. 37, no. 11, pp. 931-934, 2014.

[13] H. Yamada, T. Saito, A. Aoki et al., "Circulating betatrophin is elevated in patients with type 1 and type 2 diabetes," Endocrine Journal, vol. 62, no. 5, pp. 417-421, 2015.

[14] K. Guo, J. Lu, H. Yu et al., "Serum betatrophin concentrations are significantly increased in overweight but not in obese or type 2 diabetic individuals," Obesity, vol. 23, no. 4, pp. 793-797, 2015.

[15] X. Xie, T. Gao, M. Yang et al., "Associations of betatrophin levels with irisin in Chinese women with normal glucose tolerance," Diabetology \& Metabolic Syndrome, vol. 7, article 26, 2015.

[16] D. Moher, A. Liberati, J. Tetzlaff, D. G. Altman, and PRISMA Group, "Preferred reporting items for systematic reviews and meta-analyses: the PRISMA statement," Annals of Internal Medicine, vol. 151, no. 4, pp. 264-269, 2009. 
[17] M. Prentki and C. J. Nolan, "Islet $\beta$ cell failure in type 2 diabetes," The Journal of Clinical Investigation, vol. 116, no. 7, pp. 1802-1812, 2006.

[18] D. Giugliano, A. Ceriello, and K. Esposito, “Glucose metabolism and hyperglycemia," American Journal of Clinical Nutrition, vol. 87, no. 1, pp. 217S-222S, 2008.

[19] American Diabetes Association, "Standards of medical care in diabetes-2014," Diabetes Care, vol. 37, supplement 1, pp. S14S80, 2014.

[20] T. Ebert, S. Kralisch, A. Hoffmann et al., "Circulating angiopoietin-like protein 8 is independently associated with fasting plasma glucose and type 2 diabetes mellitus," Journal of Clinical Endocrinology and Metabolism, vol. 99, no. 12, pp. E2510-E2517, 2014.

[21] D. Espes, J. Lau, and P.-O. Carlsson, "Increased circulating levels of betatrophin in individuals with long-standing type 1 diabetes," Diabetologia, vol. 57, no. 1, pp. 50-53, 2014.

[22] Z. Fu, A. B. Abou-Samra, and R. Zhang, "An explanation for recent discrepancies in levels of human circulating betatrophin," Diabetologia, vol. 57, no. 10, pp. 2232-2234, 2014. 


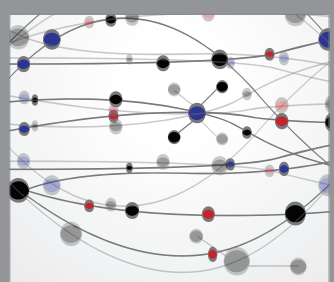

The Scientific World Journal
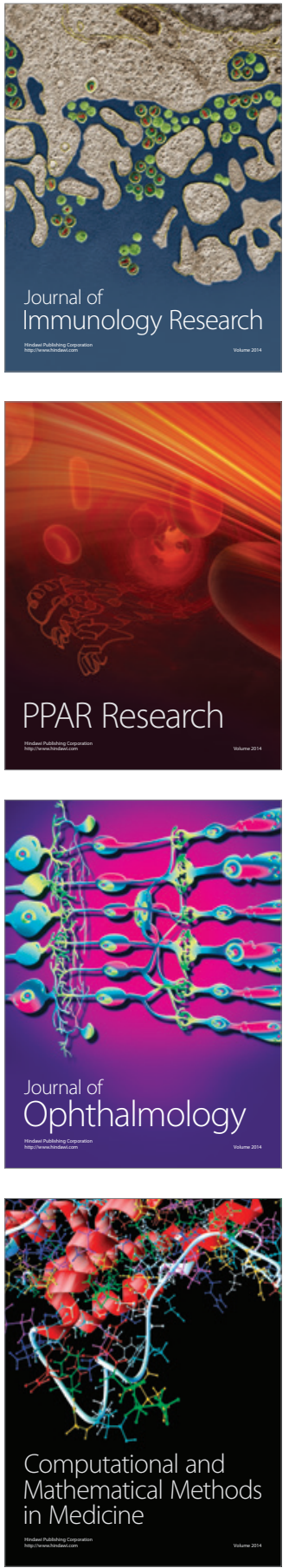

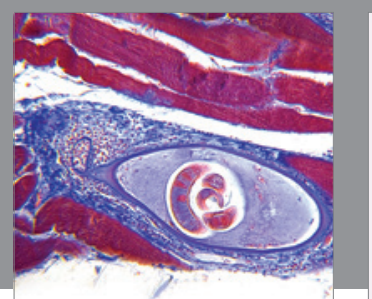

Gastroenterology Research and Practice

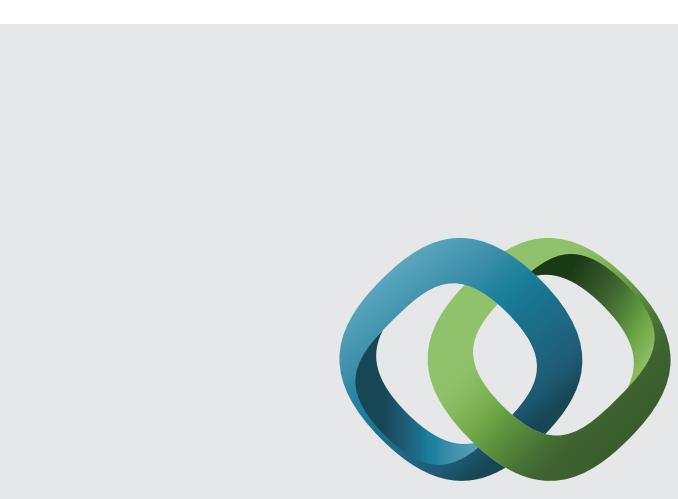

\section{Hindawi}

Submit your manuscripts at

http://www.hindawi.com
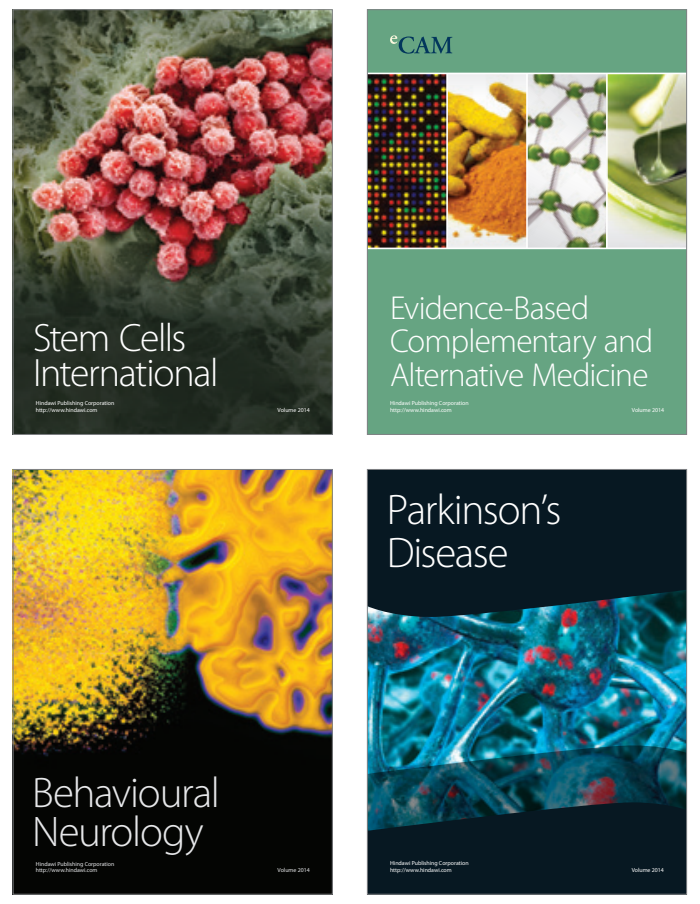
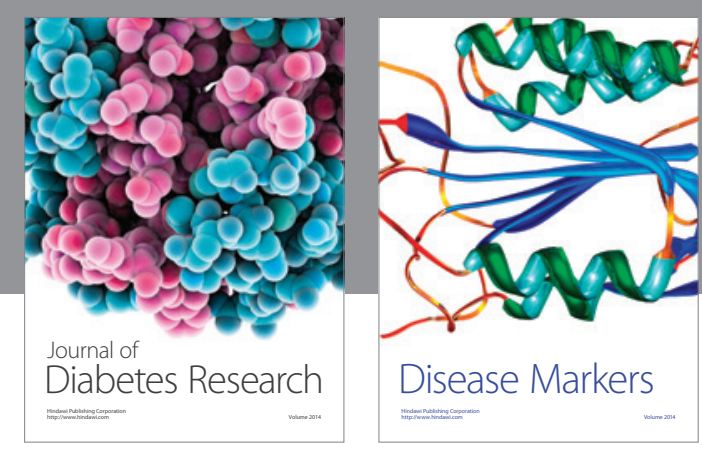

Disease Markers
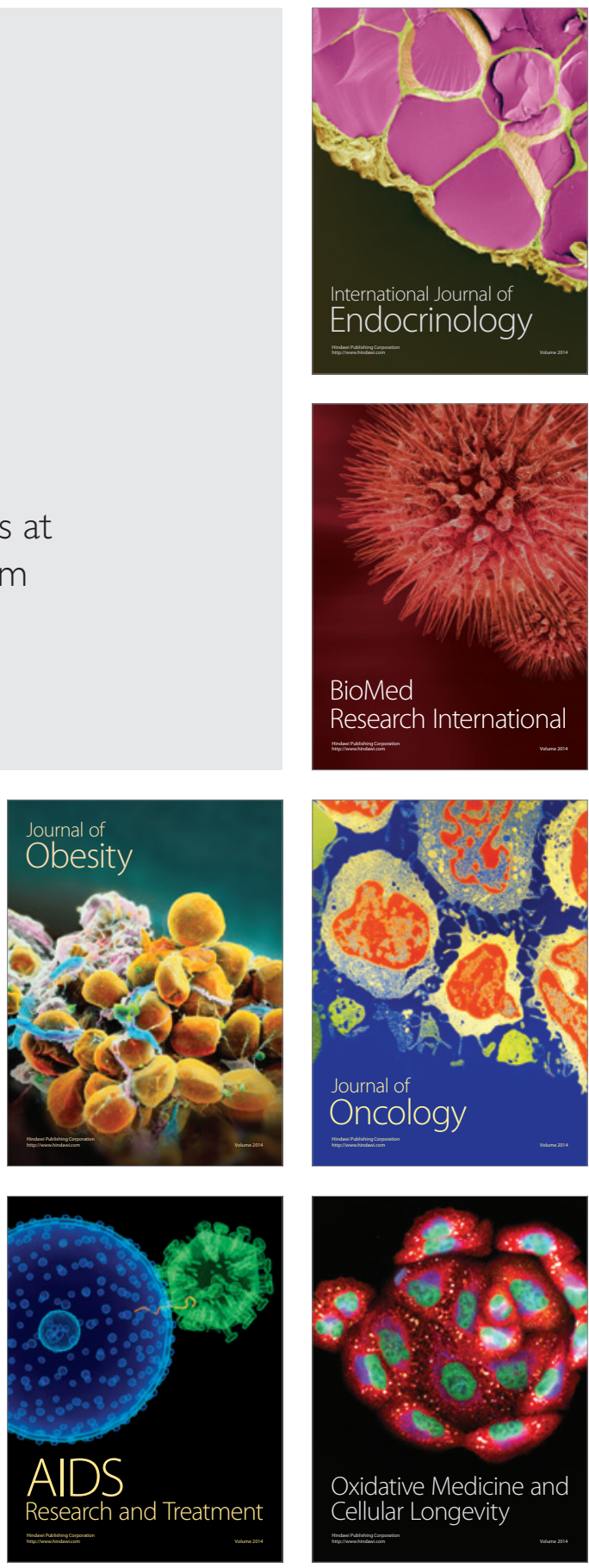